\title{
Antiferroelectric ADP doping in ferroelectric TGS crystals
}

\author{
G. Arunmozhi*, S. Lanceros-Méndez, E. de Matos Gomes \\ Departamento de Física, Universidade do Minho, Largo do Paço 4710, Braga, Portugal
}

Received 11 July 2001; accepted 28 July 2001

\begin{abstract}
Crystal growth, morphology, hysteresis and dielectric measurements on $20 \mathrm{~mol} \%$ ammonium dihydrogen phosphate (ADP)doped triglycine sulphate (TGS) crystals are reported. Crystals grew with morphology similar to phosphoric acid-doped TGS (TGSP). Inhomogeneous incorporation of dopants gives rise to a distribution in coercive fields in the different growth sectors. The incorporated dopant hinders polarization switching, which results in the increase in coercive field. No internal bias field is created by the dopant and the phase transition observed is similar to pure TGSP. The Curie point shifts to a lower temperature with increasing dopant concentration in the growth sectors. Significant changes in the activation energies of annealed specimen were identified. (c) 2002 Elsevier Science B.V. All rights reserved.
\end{abstract}

Keywords: TGS; TGSP ADP; Dielectric; Ferroelectric properties; Activation energy; Coercive fields

\section{Introduction}

Triglycine sulphate (TGS) exhibits good pyroelectric properties and finds wide application as pyroelectric detectors. TGS shows second-order phase transition at the Curie point $\mathrm{Tc}=49{ }^{\circ} \mathrm{C}$. It belongs to the monoclinic system both in the ferroelectric and paraelectric phases. Below Tc, it belongs to the polar point group 2 with the spontaneous polarization along the $b$-axis. In the paraelectric phase the crystal belongs to the centrosymmetric point group $2 / \mathrm{m} \mathrm{[1].}$ The presence of equal numbers of positive and negative domains gives rise to the macroscopic mirror

\footnotetext{
* Corresponding author. Fax: +351-53-678-981.

E-mail address: arunmg@fisica.uminho.pt (G. Arunmozhi).
}

plane at temperatures well below Curie point. The macroscopic morphology of TGS grown in the ferroelectric phase has the symmetry of nonpolar $2 / \mathrm{m} \mathrm{[2]}$. Doping with different amino acids such as L-alanine was carried out to improve the ferroelectric properties of TGS [3-8]. Doping of impurities in TGS solution results in the modification of the crystal morphology. The relative growth velocity normal to the growth axis is altered and hence the doped crystal morphology is different from the pure system [9]. In this investigation, $20 \mathrm{~mol} \%$ of ammonium dihydrogen phosphate (ADP) was doped in TGS and crystals were grown in the ferroelectric phase by a slow cooling technique. To the best knowledge of the authors, no report is available in the literature on the doping of ADP in TGS. This investigation was carried out to study the effect of antiferroelectric ADP in ferroelectric TGS. 


\section{Experimental}

\subsection{Crystal growth}

Single crystals of ADP-doped TGS were grown from aqueous TGS solution containing $20 \mathrm{~mol} \%$ of ADP. The doped solution was saturated by gradual temperature lowering and by visual observation of the convection close to a test seed suspended in the solution. Prior to actual seeding, the solution was filtered using sintered glass filter of $1-\mu \mathrm{m}$ pore size. Small seeds $(\sim 3 \mathrm{~mm})$ of TGS were suspended in the solution using a nylon thread from a stirrer, which was rotated at $20 \mathrm{rpm}$ in clockwise and counter clockwise direction. Crystals were grown by temperature lowering at a rate of $0.2-0.4{ }^{\circ} \mathrm{C}$ per day. Transparent colourless crystals weighing $15-20 \mathrm{~g}$ were grown in 30-40 days.

\subsection{Dielectric measurements}

To study the effect of ADP doping on the ferroelectric properties of TGS, dielectric measurements were performed. Small b-plates from growth pyramids (110), (001) and (010) were prepared by cleaving and cutting with a wet thread cutting machine. The surface was lapped with fine alumina powder and electronic grade silver paint was applied as electrodes. Thicknesses of the prepared samples were $1.0 \mathrm{~mm}$ and $1-2 \mathrm{~cm}^{2}$ area. Dielectric measurements were carried out using a Polymer Laboratories LCZ-meter with a programmable temperature controller coupled to a PC for data collection. The real part of the dielectric permittivity $\left(\varepsilon^{\prime}\right)$ and $\tan \delta$ were measured with a $1 \mathrm{~V} / \mathrm{cm}$ ac field at different frequencies $(300 \mathrm{~Hz}-100$ $\mathrm{kHz}$ ) and for heating cycles with the heating rate of $0.2{ }^{\circ} \mathrm{C} / \mathrm{min}$.

\subsection{Hysteresis}

Doping TGS with amino acids such as L-alanine gives rise to an internal bias field, which keeps the crystal poled at temperatures well below Tc. This is identified by the shift in the $P-E$ hysteresis loop along the $x$-axis [10]. To observe if an internal bias field is created by ADP doping, it is essential to trace the $P-E$ hysteresis loop. A conventional Sawyer-Tower circuit with a sinusoidal field of $60 \mathrm{~Hz}$ was used at room temperature on samples prepared from different growth regions. The effect of dopants on the spontaneous polarization and coercive field can be obtained from the hysteresis loops.

\subsection{X-ray diffraction}

It has been reported that the doping of TGS results in the change in lattice parameters of the doped crystal even though the concentration of the dopants estimated was lower than the concentration in the solution $[5,6]$. To detect any changes in the lattice parameters, X-ray diffraction pattern of crushed ADPTGS powder was recorded using a Philips analytical X-ray diffractometer (Model PW1710) with $\mathrm{Cu} \mathrm{K} \alpha$ radiation. The $2 \theta$ scan angle was varied from $2^{\circ}$ to $70^{\circ}$ with a step size of 0.020 .

\section{Results}

\subsection{Crystal morphology}

The crystal morphology of pure TGS was found to be modified with ADP doping. The prominent faces in TGS are $\{001\},\{101\},\{100\},\{110\},\{111\},\{121\}$, $\{021\}$ and $\{120\}$. Facets of the doped crystals were clearly identified by comparing with the morphology of pure TGS. Crystal morphology of ADPTGS and pure TGS are shown in Fig. 1.

From the morphological observation of phosphoric acid-doped TGS (TGSP), it was reported that the $\{\mathrm{hkl}\}$ and $\{\bar{h} \mathrm{k} \bar{l}\}$ faces develop in similar manner and the appearances of the $\{\mathrm{hkl}\}$ and $\{\mathrm{h} \bar{k} \mathrm{l}\}$ faces are in general identical. Based on these observations, it was concluded that the crystal morphology of TGSP shows the symmetry of $2 / \mathrm{m}$, similar to pure TGS [9]. Since the crystal morphology of ADPTGS is similar to TGSP morphology, it can be concluded that ADPTGS also shows the symmetry $2 / \mathrm{m}$. The nonpolar symmetry observed in the crystal morphology is associated with the multidomain structure [9].

The $\{001\}$ faces were prominent in pure and doped TGS crystals; $\{010\}$ faces, which hardly developed in TGS, grew wider in ADPTGS. The $\{120\},\{021\}$ faces surrounding $\{010\}$ in pure TGS disappear in ADPTGS due to the substantial development of $\{010\}$. Also, $\{11 \overline{1}\},\{\overline{1} 21\}$ faces were less developed. 


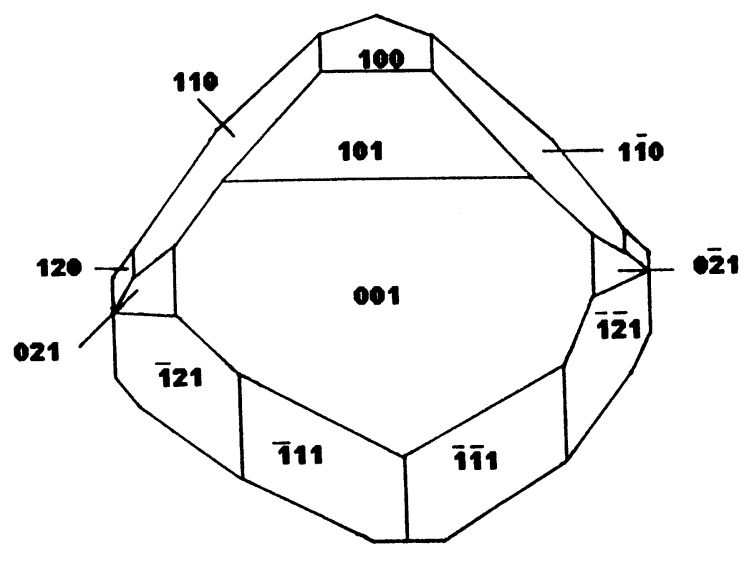

(a)

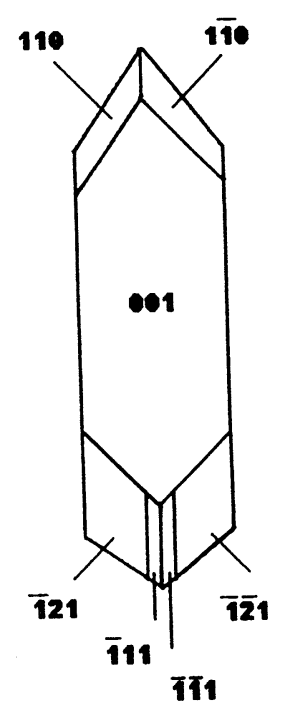

(b)

Fig. 1. Crystal morphology of (a) pure TGS (b) ADPTGS.

While the $\{110\}$ faces were less affected by doping, $\{101\}$ and $\{100\}$ faces, which develop in pure TGS and phosphoric acid-doped TGS, disappeared in ADPTGS.

Development of facets depends on the reduction in the growth velocity normal to the plane and impurities in the growth solution also influence the crystal growth kinetics. In pure TGS the $\{010\}$ face hardly develops. With doping, growth along the polar axis is severely restricted and the doped crystals exhibit well developed $\{010\}$ face. It is conceivable that large amount of dopant enters into the lattice normal to this plane. Such an inhomogeneous incorporation of dopant was observed in TGS doped with phosphoric acid [9].

It has been found that the dopant concentration in the solution $\left(x_{\mathrm{s}}\right)$ is far higher than the concentration in the crystal $\left(x_{\mathrm{c}}\right)$. In TGS crystals grown with different concentrations of phosphate, it was reported that the ratio of $x_{\mathrm{c}} / x_{\mathrm{s}}$ at the maximum is $10^{-2}$. The magnitude of coercive field $\left(E_{\mathrm{c}}\right)$ increases with $x_{\mathrm{c}}$. Hence, from $E_{\mathrm{c}}$ the dopant concentration in the crystal can be estimated [11]. Assuming that phosphate has entered into the lattice, the mole fraction of the dopant concentration for ADPTGS crystals obtained from Ec values were found to be $0.7 \times 10^{-3}$, which corresponds to TGS with $30 \mathrm{~mol} \%$ of phosphate. However, the crystal morphology of ADPTGS is found to be similar to TGS with $50 \mathrm{~mol} \%$ of phosphate doping. It is not clear at this stage if phosphate group of ADP alone enters the lattice. Even at very low dopant incorporation, significant changes were observed in the ferroelectric properties similar to the ones reported on doped TGS [8].

\subsection{X-ray diffraction}

The X-ray diffraction pattern of ADP-doped TGS was identical to that of pure TGS and hence no change in the lattice parameters could be detected. Similar results were obtained in phosphoric aciddoped TGS [9].

\subsection{Dielectric studies}

Dielectric measurements were recorded on (1) samples that did not go through any thermal history 


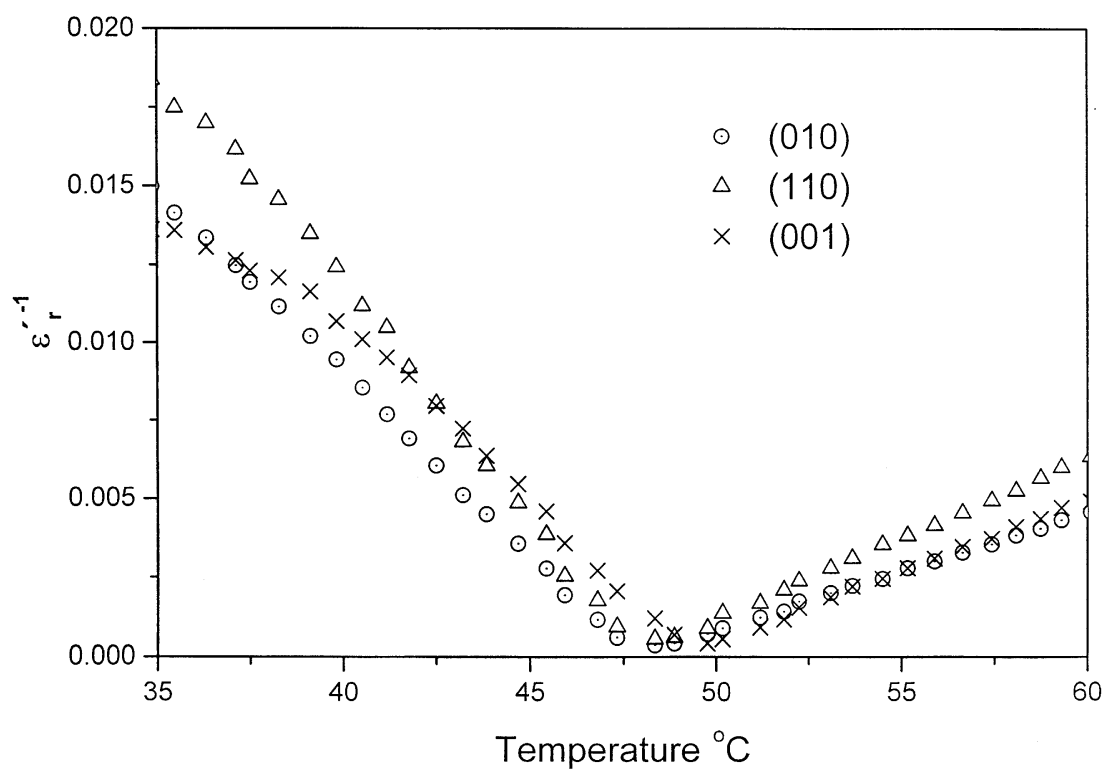

Fig. 2. Temperature dependence of $1 / \varepsilon_{\mathrm{r}}^{\prime}$ measured in the different growth sectors at $v=1 \mathrm{kHz}$.

(unannealed), and (2) samples that were preheated at a slow heating rate $\left(0.2{ }^{\circ} \mathrm{C} / \mathrm{min}\right)$ through the transition temperature, and cooled to room temperature before the actual experiments in order to remove any residual strains and to change the domain structure in the crystal (annealed).

Temperature dependence of the inverse dielectric permittivity of the different growth sectors (the so called growth pyramids) in ADPTGS measured at 1 $\mathrm{kHz}$ is shown in Fig. 2.

The dependence of dielectric permittivity on temperature and frequency is similar to TGSP. The decrease in the permittivity of pure TGS $\left(\varepsilon^{\prime}=7000\right)$ due to ADP doping $\left(\varepsilon^{\prime}=3000\right)$ was identified. The decrease in the dielectric permittivity in ADPTGS from pure TGS can be ascribed to the clamping of domains and pinning of dipoles by the dopant, and the availability of extra protons for charge compensation due to the phosphate substitution in the lattice. No distinct change in the ferroelectric nature of phase transition could be observed in the two types of samples due to the doping of antiferroelectric ADP. However, a shift in the Tc to a lower point has been found to increase with increasing coercive fields in the growth sectors, which agree well with the dielectric studies on TGSP [12]. The reduced shift of the Curie point has been shown to be equal to the mole fraction of the dopant in the crystal [12]. Even within the growth pyramids, the distribution is not uniform. There exists a distinct relation between the coercive field and the shift in Tc value. The distribution of coercive field in the growth pyramids increases in the order $(010)>(110)>(001)$ and the shift in Tc is in the order $(010)<(110)<(001)$. The shift in the Tc values indicates that the distribution of dopants leads to micro regions in the crystal with different transition temperatures. The results are presented in Table 1. The distribution of impurities in the crystal contribute for

Table 1

Variation of Curie point $(\mathrm{Tc})$, Curie constant in the paraelectric $\left(C_{\mathrm{p}}\right)$ and ferroelectric $\left(C_{\mathrm{f}}\right)$ values for the different growth sectors

\begin{tabular}{|c|c|c|c|c|c|c|c|}
\hline \multirow[t]{2}{*}{$\begin{array}{l}\text { Growth } \\
\text { sector }\end{array}$} & \multirow[t]{2}{*}{$\begin{array}{l}\mathrm{Tc} \\
\left({ }^{\circ} \mathrm{C}\right)\end{array}$} & $\begin{array}{l}C_{\mathrm{p}} \\
(\mathrm{K})\end{array}$ & $\begin{array}{l}C_{\mathrm{f}} \\
(\mathrm{K})\end{array}$ & $\begin{array}{l}C_{\mathrm{p}} / \\
C_{\mathrm{f}}\end{array}$ & $\begin{array}{l}C_{\mathrm{p}} \\
(\mathrm{K})\end{array}$ & $\begin{array}{l}C_{\mathrm{f}} \\
(\mathrm{K})\end{array}$ & $\begin{array}{l}C_{\mathrm{p}} / \\
C_{\mathrm{f}}\end{array}$ \\
\hline & & \multicolumn{3}{|c|}{ Unannealed } & \multicolumn{3}{|c|}{ Annealed } \\
\hline (010) & 48.3 & 2685 & 930 & 2.89 & 2654 & 835 & 3.18 \\
\hline (110) & 48.4 & 2165 & 835 & 2.59 & 1974 & 575 & 3.44 \\
\hline (001) & 49.3 & 2439 & 1117 & 2.18 & 2348 & 1000 & 2.34 \\
\hline
\end{tabular}


the rounding effect observed near the Curie point. This has been demonstrated by the measurements of different coercive fields in the different growth sectors.

The ratio of the Curie constants in the paraelectric phase $\left(C_{\mathrm{p}}\right)$ and in the ferroelectric phase $\left(C_{\mathrm{f}}\right)$ show a marginal increase in the values for annealed samples. According to thermodynamic theory, increase in the ratio $\left(C_{\mathrm{p}} / C_{\mathrm{f}}\right)=2$ is due to the contributions from the clamping and adiabatic effects. In the ferroelectric phase, domain wall motions contribute to the dielectric constant at temperature below Tc. For ADPTGS, the domain motion is restricted by the dopants and hence the contribution of the domain wall motion in the ferroelectric phase is much lower. This results in very low $\left(C_{\mathrm{f}}\right)$ values.

For unannealed samples from the (110) growth sector, the $\tan \delta(T, v)$ spectra show a broadened maxima, which reaches a minimum value around $40{ }^{\circ} \mathrm{C}$. For annealed samples from the same growth sector, the maxima in $\tan \delta$ disappears as shown in Fig. 3. Above Tc, shifts in the $\tan \delta(T, v)$ show that the domain walls still retain the thermal memory. The coercive fields of samples from this growth sector are found to be high ( $>90 \mathrm{kV} / \mathrm{m})$.

The $\tan \delta(T, v)$ spectra for samples from the growth sector (010) are shown in Fig. 4. The tan $\delta$ spectra for the unannealed samples show a similar trend as the fresh samples from the (110) growth sector. However, the broadened maxima extends till the transition temperature and is also observed on annealed samples. The broadened maxima indicates the clamping of the domains, which actively take part in the dynamics. A high concentration of dopants enters normally to this growth sector, which causes the broad maxima to extend till Tc.

In TGS, the majority of the domains are found in the form of lenticular shape with the major axes roughly perpendicular to the $c$-axis. With the samples annealed, the lenticular domains turn into fine lamellar structure. The direction of the lamellas is parallel to the long axis of the lenticular domains [13]. The distinct change in the $\tan \delta$ for annealed samples can

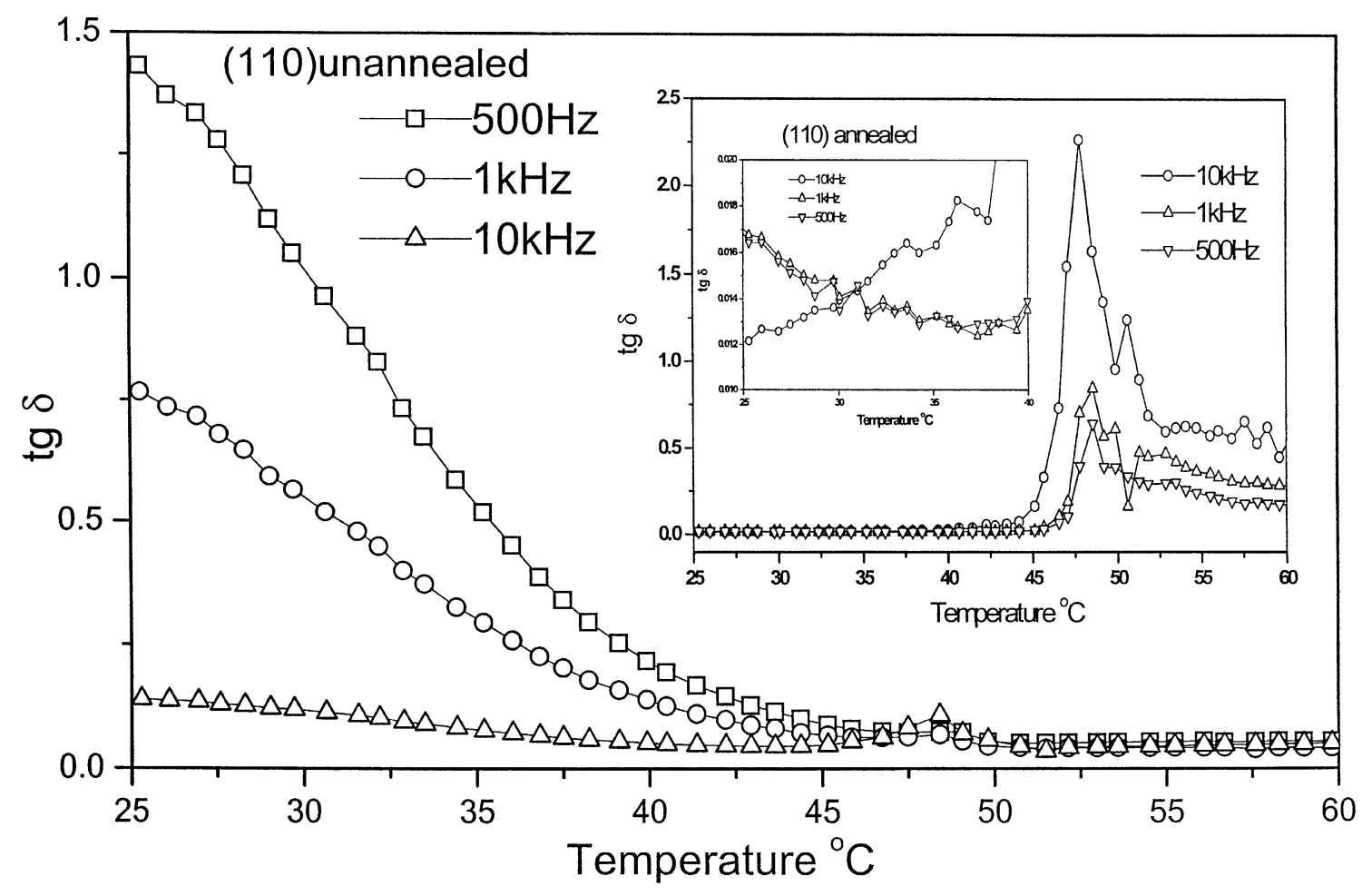

Fig. 3. Dependence of $\operatorname{tg} \delta(T, \gamma)$ in the (110) growth sector for unannealed and annealed (inserted figure) specimens. 


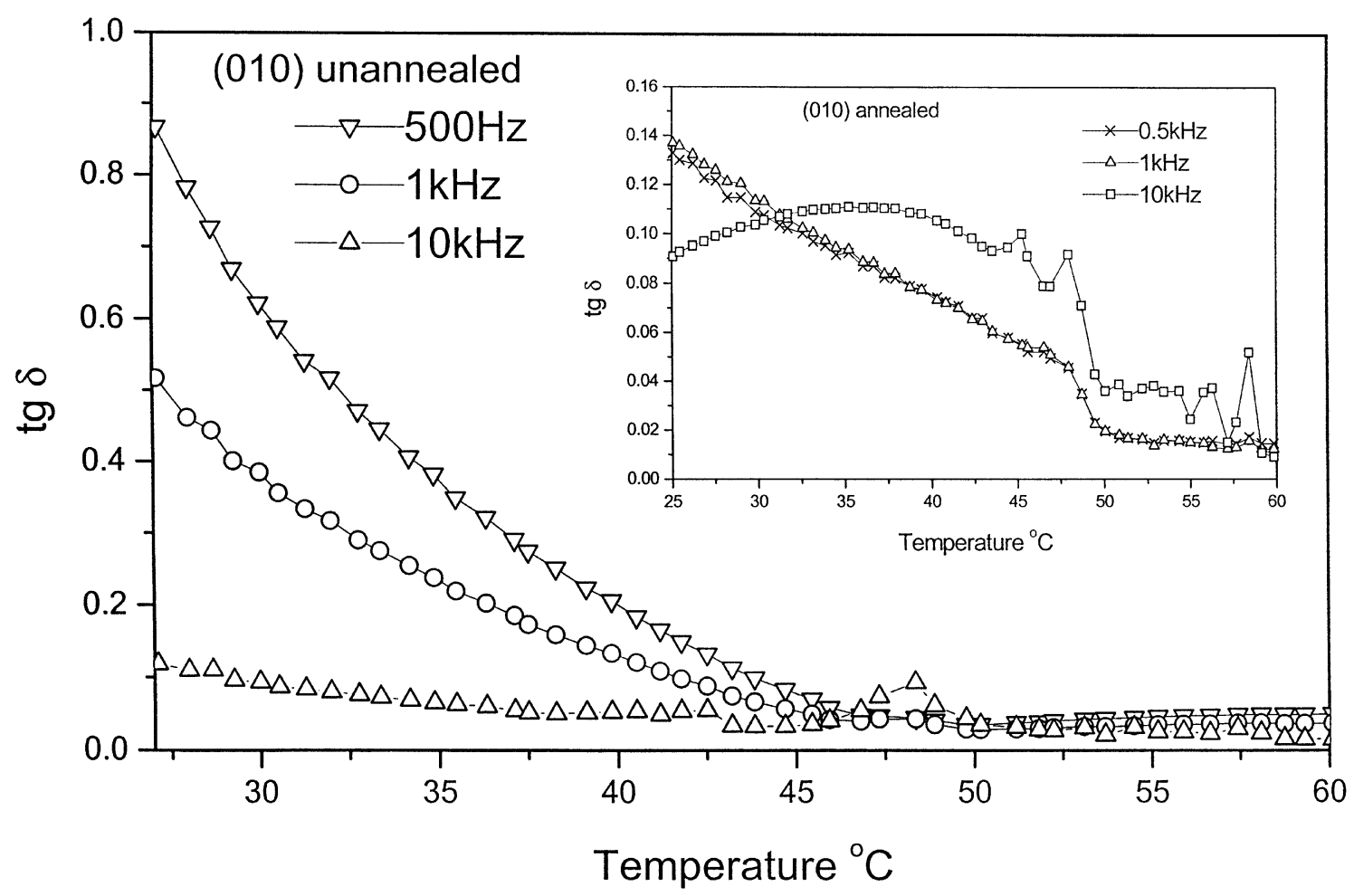

Fig. 4. Dependence of $\tan \delta(T, \gamma)$ in the (010) growth sector for unannealed and annealed (inserted figure) specimens.

be related to the crystallographic orientation of the domain structure.

The structure of TGS is made up of three glycine molecules, namely, GI, GII and GIII. The ferroelectric nature of TGS arises due to the GI glycine molecule. In the ferroelectric phase, the $\mathrm{CH}_{2}-\mathrm{NH}_{3}$ and $\mathrm{NH}_{3}$ groups of GI molecule undergo flipping and rotation motions. Rotation of $\mathrm{NH}_{3}$ group of GII and GIII also occurs. The dielectric dispersion observed in the range $270-300 \mathrm{~K}$ on L-threonine-admixtured TGS (LTTGS) was shown to be due to a combination of the hindered rotation of the $\mathrm{NH}_{3}$ groups of GII and GIII glycines and domain structure.

From the present experiments on ADPTGS, it is found that the maximum contribution to the dielectric dispersion comes from the domain structure. With the samples heated through the transition temperature and cooled down to room temperature, the domain structure becomes fine thin strips (lamellar structure) [13]. The concentration of dopant is very low in the (001) growth sector, hence less dipoles are restricted, and the maxima in the $\tan \delta$ spectra of this growth sector occurs at temperatures less than $35{ }^{\circ} \mathrm{C}$, which agrees well with the results shown on LTTGS [14] (Fig. 5). A distinct kink is observed around $32{ }^{\circ} \mathrm{C}$ in the (001) growth sector (unannealed), which decreases with decreasing frequencies, disappearing at low frequencies $(200 \mathrm{~Hz})$ and by annealing the sample. This is related to the strains created by the dopant and is found to be removed by annealing. The low coercive field substantiates that less dipoles are restricted in this growth sector.

\subsection{Activation energy}

The activation energies were calculated based on the Arrhenius equation $\tau=\tau_{\mathrm{o}} \exp -E_{\mathrm{a}} / k T$, where $\tau$ is the relaxation time, $\tau_{\mathrm{o}}$ the pre-exponential factor, $E_{\mathrm{a}}$ the activation energy, $k$ the Boltzman constant, and $T$ 


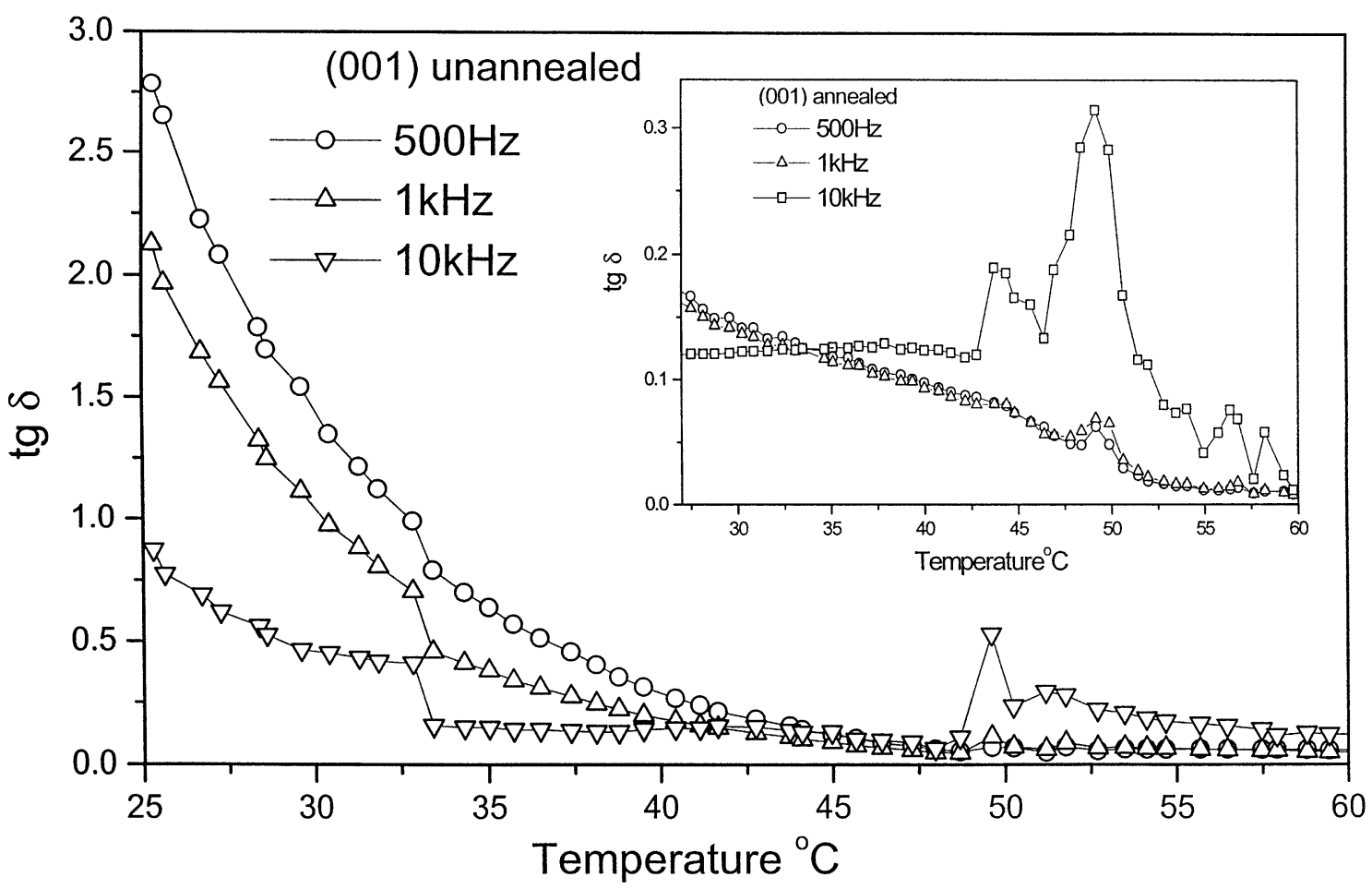

Fig. 5. Dependence of $\tan \delta(T, \gamma)$ in the (001) growth sector for unannealed and annealed (inserted figure) specimens.

the temperature in $\mathrm{K}$. The results for the two kinds of samples are given in Table 2.

A sharp decrease in the activation energies for annealed samples shows that the change in the domain structure plays a significant role in the relaxation process. Large drop in the activation energy between the two kinds of samples was clearly evident in the samples cut in the (110) growth sector. Domain reversal in the (010) growth sector is more strained than in the other two growth sectors as is evident from the activation energies.

Table 2

Activation energies for unannealed and annealed specimen from the three growth sectors

\begin{tabular}{lll}
\hline $\begin{array}{l}\text { Growth } \\
\text { sector }\end{array}$ & $\begin{array}{l}E_{\mathrm{a}}(\mathrm{eV}), \\
\text { unannealed }\end{array}$ & $\begin{array}{l}E_{\mathrm{a}}(\mathrm{eV}), \\
\text { annealed }\end{array}$ \\
\hline$(010)$ & 2.11 & 1.60 \\
$(110)$ & 3.29 & 0.45 \\
$(001)$ & 1.35 & 0.74 \\
\hline
\end{tabular}

\subsection{Hysteresis}

From the $P-E$ hysteresis loops, the spontaneous polarization and coercive fields in the different growth sectors were measured and are shown in Table 3.

There is no distinct change in the spontaneous polarization due to ADP doping in TGS. The absence of internal bias field shows that the dopant creates nonpolar effects in the lattice. Due to ADP doping in

Table 3

Coercive field $\left(E_{\mathrm{c}}\right)$, spontaneous polarization $\left(P_{\mathrm{s}}\right)$ obtained from hysteresis for TGS and ADPTGS

\begin{tabular}{lcll}
\hline & $\begin{array}{l}\text { Coercive } \\
\text { field } \\
(\mathrm{V} / \mathrm{cm})\end{array}$ & $\begin{array}{l}\text { Spontaneous } \\
\text { polarization } \\
\left(\mu \mathrm{C} / \mathrm{cm}^{2}\right)\end{array}$ & $\begin{array}{l}\text { Growth } \\
\text { sector }\end{array}$ \\
\hline TGS & 370 & 2.8 & $(010)$ \\
ADP + & 3250 & 3.0 & $(010)$ \\
TGS & 930 & 3.2 & $(110)$ \\
\hline
\end{tabular}


TGS, only an increase in the coercive field is observed in the different growth sectors.

\section{Conclusion}

The morphology of the ADPTGS crystals is similar to TGSP and is interpreted that significant amount of phosphate is incorporated in the lattice. The presence of amino group in ADP similar to the amino group in glycine, is presumed to enhance the dopant incorporation in the pure lattice. Since no change in the nature of ferroelectric phase transition is observed in ADPTGS, it can be concluded that the antiferroelectric properties of ADP do not have an influence on the ferroelectric properties of TGS and ADP acts more like an impurity or dopant. Like in TGSP, no internal bias field is created and hence nonpolar effects are generated with ADP doping. Increase in the coercive field values in ADPTGS shows that the dopant restricts dipoles from switching. Dielectric dispersion above room temperature and near the Curie point is defined by the domain structure, and the maxima observed in the loss spectra of ADPTGS shows the clamping of domains. This is also evident from the increase in the $C_{\mathrm{p}} / C_{\mathrm{f}}$ values. Shift in Tc shows that inhomogeneous dopant incorporation leads to micro regions in the crystal growth sectors with different transition temperatures.

\section{Acknowledgements}

The authors are grateful to the Institute of Materials (IMAT), Universidade do Minho for the financial assistance to carry out this project.

\section{References}

[1] Landolt-Bornstein, Crystal and Solid State Physics, New series, Group III, vol. 16b, Springer, New York, p. 223.

[2] B. Brezina, M. Havrankova, Cryst. Res. Technol. 20 (1985) 787.

[3] W. Wang, C.S. Fang, Cryst. Res. Technol. 27 (1992) 245.

[4] Y. Kim, G. Park, Ferroelectrics 146 (1993) 99.

[5] G. Ravi, S. Anbukumar, P. Ramasamy, Mater. Chem. Phys. 37 (1994) 180.

[6] G. Arunmozhi, R. Jayavel, C. Subramanian, Mater. Chem. Phys. 50 (1997) 57.

[7] G. Arunmozhi, R. Jayavel, C. Subramanian, Mater. Lett. 33 (1998) 251.

[8] C.S. Fang, X.I. Yao, A.S. Bhalla, L.E. Cross, Ferroelectrics 51 (1983) 9.

[9] N. Nakatani, M. Yoshio, Jpn. J. Appl. Phys. 35 (1996) L508.

[10] N. Nakatani, Jpn. J. Appl. Phys. 30 (1991) 1024.

[11] N. Nakatani, M. Yoshio, Jpn. J. Appl. Phys. 35 (1996) 5752.

[12] N. Nakatani, M. Yoshio, Jpn. J. Appl. Phys. 36 (1997) L425.

[13] N. Nakatani, Jpn. J. Appl. Phys. 12 (1973) 1723.

[14] A. Czarnecka, J. Stankowska, Phys. Status Solidi B 207 (1998) 557. 